\title{
Detección y representación de eventos ambientales basada en agentes
}

\section{Detection and representation of environment events based in intelligent agents}

\author{
PADILLA-CUEVAS, Josué †*, REYES-ORTIZ, José A., BRAVO, Maricela y GARCÍA-ROBLEDO, \\ Gabriela A.
}

Universidad Autónoma Metropolitana Unidad Azcapotzalco, Departamento de Sistemas

ID $1^{\mathrm{er}}$ Autor: Josué, Padilla-Cuevas ORC ID: 0000-0002-3156-3231, CVU CONACYT ID: 809289

ID $1^{\mathrm{er}}$ Coautor: José A., Reyes-Ortiz / ORC ID: 0000-0003-2111-4982, CVU CONACYT ID: 207359, Researcher ID Thomson: 57188692429, CVU CONACYT ID: 63521

ID $2^{\text {do }}$ Coautor: Maricela, Bravo / ORC ID: 0000-0003-3493-9131, CVU CONACYT ID: 97758 , Researcher ID Thomson: 14037071900

ID $3^{\text {er }}$ Coautor: Gabriela A., García-Robledo / ORC ID: 0000-0002-4559-651X, CVU CONACYT ID: 881294

DOI: $10.35429 / J T E N .2019 .12 .3 .16 .25$

Recibido 20 de Octubre, 2019, Aceptado, 19 de Diciembre, 2019

\section{Resumen}

Un Ambiente inteligente puede responder a las necesidades de los usuarios según el contexto, esto para que los individuos tengan las condiciones climáticas ideales para poder llevar a cabo sus actividades, estas condiciones están reguladas por una serie de normas especiales. Los eventos que se describen en este artículo están relacionados con sucesos del medio ambiente (temperatura, humedad, luminosidad y presencia), además envuelven diferentes variables como tiempo, espacio o persona, porque son importantes para modelar lo que está ocurriendo en un determinado lugar. En este trabajo, se propone un diseño ontológico personalizado para el dominio académico. El modelo ontológico es utilizado para la identificación de eventos ambientales a partir de los datos adquiridos del entorno mediante la simulación de agentes inteligentes. Además, el modelo ontológico se usa para razonar con la información obtenida de los eventos identificados. El modelo de ontologías basado en eventos considera cuatro cuestiones contextuales como una perspectiva modular: persona, temporalidad (tiempo), espacialidad (ubicación), red (recursos para adquirir datos del ambiente) y evento (eventos académicos). Y el detector se fundamenta en reglas obtenidas de los estándares de las condiciones climáticas óptimas de un espacio físico.

\begin{abstract}
An Intelligent environment can respond to the necessities of the users according to the context, this is so that the individuals can have the ideal climatic conditions in order to go about their activities, these conditions are related through a series of special norms. The events that we describe in this paper are in relation to the events of the environment (temperature, humidity, brightness, and presence), also involved are different variables like time, space, or person, such are important in order to be able to model what is occurring in a determined place. In this project, we propose a personalized ontological design for the academic dominion. The ontological model is utilized for the identification of environmental events according to the data acquired from the environment through the simulation of intellectual agents. Also, our ontological model is used to rationalize with the information obtained from the identified events. The model of ontologies based on events considers four contextual questions like a perspective modular: person, seasonality (weather), spatiality (location), network (resources in order to acquire environmental data) and event (academic events). And the detector is based on rules obtained from the standards of optimum climatic conditions of a physical space.
\end{abstract}

Ontología, Agentes y Eventos

Ontology, Agents, Events

Citación: PADILLA-CUEVAS, Josué, REYES-ORTIZ, José A., BRAVO, Maricela y GARCÍA-ROBLEDO, Gabriela A. Detección y representación de eventos ambientales basada en agentes. Revista de Ingeniería Tecnológica. 2019 3-12: 16-25

\footnotetext{
* Correspondencia del Autor (Correo electrónico: jpc@azc.uam.mx)

$\dagger$ Investigador contribuyendo como primer autor.
} 


\section{Introducción}

En un ambiente académico se realizan diariamente con alta frecuencia actividades de docencia, tales como: impartir asesorías, impartir clases, dirigir prácticas de laboratorio. También existen actividades de investigación: redactar artículos, lectura de artículos, dirigir experimentos científicos. Finalmente, diversas actividades de difusión suceden en un espacio académico, como: seminarios, conferencias, reuniones de proyectos de investigación.

Para poder realizar estas actividades es necesario que los participantes de un ambiente académico cuenten con las condiciones climáticas ideales. Y un ambiente sensible al contexto puede proporcionar esas condiciones de manera automática para los usuarios. Por ejemplo, al tener la luminosidad adecuada dentro de un salón de clases, se maximiza la utilidad de los recursos eléctricos mediante regular las lámparas y encender los proyectores automáticamente solo cuando sea necesario.

Es por ello que la detección de eventos ambientales se vuelve un proceso fundamental dentro de un ambiente académico inteligente.

La detección de eventos consiste en que el sistema sea capaz de reaccionar a los eventos y a las condiciones del lugar despreciando su naturaleza, por ejemplo, entradas y salidas de personas, variaciones de temperatura, humedad e iluminación, eventos que son detectados y almacenados para su uso posterior.

Por su parte, la representación de eventos consiste en almacenar información sobre lo que sucede o los hechos acaecidos en el ambiente académico inteligente, mientras que las ontologías son herramientas que sirven para estructurar conceptualmente determinados ámbitos del conocimiento por medio de vocabularios controlados, proporcionando la información para la construcción del sistema todo con el fin de aportar consistencia, fiabilidad. Además, en este artículo se decidió utilizar ontologías para agregar semántica al esquema.

El objetivo principal de este trabajo es diseñar, implementar y evaluar un sistema para la detección y representación semántica de eventos ambientales en un ambiente académico usando ontologías, con la finalidad de ofrecer servicios a los usuarios de manera inteligente.
Conjuntamente, las ontologías generan un beneficio porque van a permitir representar los conceptos para detectar e inferir sobre eventos simples relacionados con el medio ambiente.

Este documento está dividido en 6 secciones. En la sección 2 expone los trabajos donde se muestran los avances más importantes que se han logrado con respecto al conocimiento en el área de investigación de este trabajo. En la sección 3, se presenta el sistema ontológico adaptado al dominio académico con el fin de respaldar y representar los eventos ambientales detectados, dicho modelo se basa en el uso de ontologías modulares. La sección 4 muestra el proceso de detección de eventos, los módulos creados y la simulación de datos obtenida por agentes inteligentes. La sección 5 discute la evaluación del modelo ontológico obtenido por medio de casos de uso. Además, se utilizan las métricas conocidas de precisión y exhaustividad para la evaluación del sistema de detección de eventos. Por último, se exponen las conclusiones generales de este trabajo y se presentan las propuestas para trabajos a futuro.

\section{Trabajos relacionados}

En esta sección se presenta una revisión del estado del arte en el tema de la detección de eventos en distintos ámbitos o dominios, así como las herramientas utilizadas para su representación. Existen diversos trabajos que se fundamentan en ontologías para la detección de eventos en distintos ámbitos, por ejemplo, el automotriz o el de la salud, pero pocos han aplicado éstas herramientas para la detección de eventos ambientales en un contexto académico.

Pavle Skocir, Petar Krivic y Gordan Jezic [1] reportan en un artículo del 2016, un análisis de datos en un ambiente inteligente, que se centra solamente en la detección de entradas y salidas desde una habitación. Georgios Meditskos Ioannis Kompatsiaris [2] crean iKnow.

Es un modelo basado en una ontología para la comprensión del contexto en ambientes con múltiples sensores que son utilizados para el reconocimiento de la actividad humana. IKnow utiliza el uso del conocimiento ontológico OWL para capturar relaciones de dominio entre observaciones y actividades. 
La propuesta de Liming Chen, Chris Nugent [3] describe un modelo basado en ontologías para el reconocimiento de actividades. El enfoque propuesto adopta ontologías para el modelado de sensores, objetos y actividades, además explota el razonamiento lógico para los propósitos de la actividad de reconocimiento en una casa inteligente, utilizan algoritmos de reconocimiento de actividad, incluyendo los métodos de aprendizaje supervisados y no supervisados, con un razonamiento probabilístico y estadístico.

Otro trabajo relacionado a la especificación de detección de eventos y representación mediante ontologías es el de Jean-Paul Calbimonte, Jean-Eudes Ranviera, Fabien Dubosson, Karl Aberer [4] que presentan un framework para inferir eventos semánticamente de glucosa obtenidos de pacientes, mediante datos de sensores móviles desplegados en un cinturón deportivo. Este trabajo es parte del proyecto D1namo para monitoreo de la diabetes, se centra en la representación y el procesamiento de consultas de datos producidos por los sensores portátiles, utilizando tecnologías semánticas. Además, utiliza un motor de procesamiento de flujo RDF.

Finalmente, Markus Stocker, Mauno Rönkkö, and Mikko Kolehmainen [5]. Presentan en junio de 2014 un modelo de software que representa una máquina de aprendizaje para el monitoreo de un ambiente, utilizando sensores y representando el conocimiento de los eventos adquiridos, o extraídos, en módulos de aprendizaje.

Su funcionalidad es la evaluación de la situación, es decir. Para ganar conciencia de la situación. Se basa en la representación del conocimiento y el razonamiento, utilizando ontologías, es utilizado para el aprendizaje del movimiento de los vehículos que pasan sobre una carretera y utiliza Weka para el aprendizaje automático.

\section{Modelo de representación de eventos ambientales}

Según Barranco [6], los eventos son definidos como la ocurrencia dentro de un sistema o dominio particular. De acuerdo con Miller [7] un evento se define como un suceso que involucra un cambio de estado, donde se involucran aspectos locativos, temporales y causales.
Allen [8], expone que el mundo contiene eventos que son el camino por el cual los agentes clasifican ciertos patrones de cambio y finalmente Sowa [9], afirma que un evento es una entidad que puede involucrase en la causalidad y que puede ser identificado por su ubicación en una región del espacio-tiempo.

En esta sección se presenta el desarrollo del modelo ontológico que almacena los eventos ambientales que ocurren dentro de un espacio académico, el modelo consta de cinco ontologías modulares, que son: tiempo, espacio, persona, red se sensores y evento.

Tienen el propósito de representar donde ocurrió un evento, el instante cuando ocurrió y finalmente las personas involucradas.

Estas ontologías modulares se unifican en un solo modelo semántico llamado "Ambiente Inteligente".

Según Gruber [10] y Guarino [11], "una ontología es una descripción formal de los conceptos y las relaciones entre ellos". Por lo tanto, las ontologías proporcionan el mecanismo para representar conceptos formalmente, además son comprensibles por los humanos y procesables para las computadoras.

\section{Ontología de espacio físico}

La ontología de espacio físico representa el lugar donde diariamente ocurren actividades académicas, son lugares donde existen estructuras e instalaciones necesarios para el funcionamiento de una organización en este caso académica, con este modelo se puede saber en dónde están ocurriendo los eventos ambientales dentro de un espacio académico.

La ontología Figura 1 está dividida en espacios cerrados que se encuentran delimitados por divisiones físicas en estos se encuentran los cubículos, laboratorios, aulas, auditorios, oficinas administrativas, auditorios, edificios, salones de clase, comedores y baños.

Los espacios abiertos son aquellos que carecen de una estructura física y que son afectados por el medio ambiente. Están divididos en: corredores o pasillos, áreas verdes, estacionamientos y plazas. 
La ontología de espacio físico tiene un total de 13 propiedades de datos: hasAirConditioner, hasArea, hasCar-Capacity, hasDoorState, hasFan, hasLampsNumber, hasLevel, hasNamePhysicalSpace, hasPeopleCapacity, hasProjectionScreen, hasProjector, hasService y hasWindow.

Se utilizan para modelar a detalle los servicios que se encuentran dentro de un espacio determinado y al lograr manipularlos obtener un ambiente sensible al contexto.

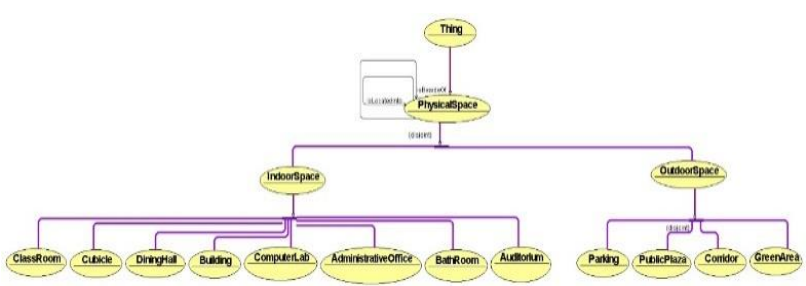

Figura 1 Ontología de espacio físico

\section{Ontología de tiempo}

El modelado del tiempo en una ontología es de propósito general, es decir, es una ontología de nivel alto que describe un concepto muy frecuentemente requerido, en este caso el tiempo, permite definir eventos $y$ correlacionarlos con el instante cuando han ocurrido los eventos en el ambiente académico.

La ontología de tiempo Figura 2 está compuesta por dos clases que son, intervalo que a su vez se componen de instantes, uno para indicar cuando inicia el evento, este se representa con la propiedad de objeto hasBeggining y el segundo intervalo se utiliza para determinar cuándo termina el evento: hasEnd.

Las propiedades de datos sirven para representar las unidades básicas de tiempo como son: horas, minutos, segundos, año, mes y día.

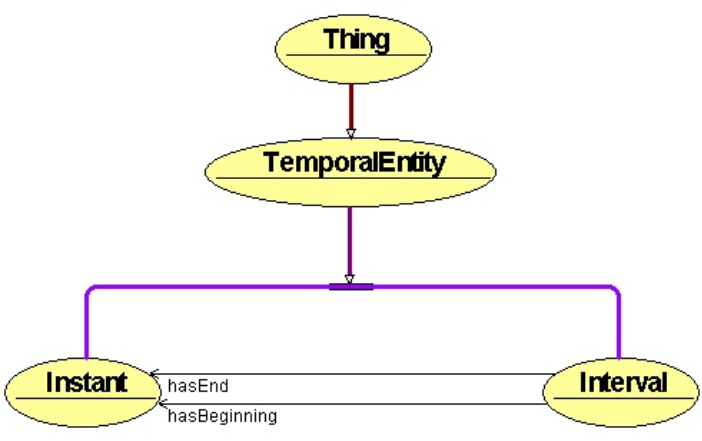

Figura 2 Ontología de tiempo

\section{Ontología de persona y red de sensores}

Un elemento fundamental del modelado de eventos es la representación o identificación del actor que participa o al que le sucede un evento. Sin la representación de los actores en el evento no se tendría el conocimiento de quien está involucrado dentro de un acontecimiento. Implementar un modelo completo para la representación de personas puede llegar a ser demasiado extenso, es por eso que en este trabajo se ha acotado a un modelo (Figura 3) donde solamente se representan los actores que participan dentro del dominio académico, como son: estudiantes, empleados tanto administrativos como profesores y visitantes que también tienen un rol importante dentro de este ambiente académico [12].

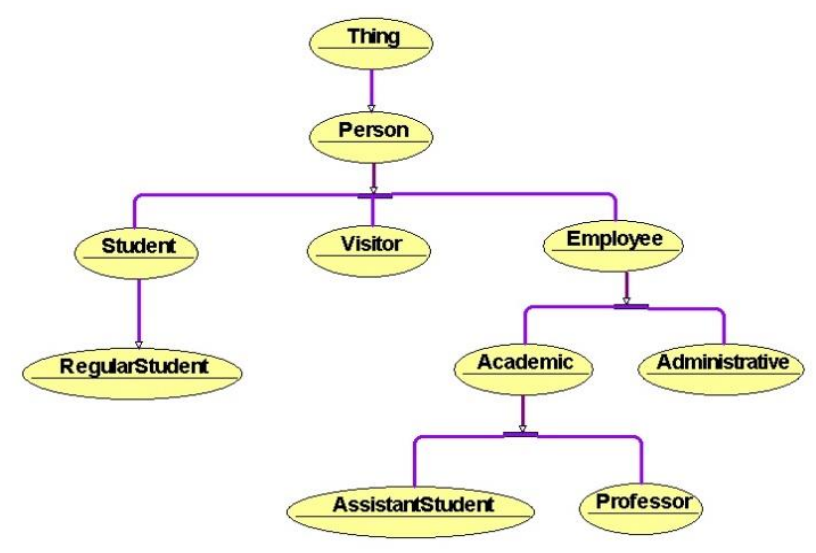

Figura 3 Ontología de persona

Entre la información más relevante dentro de un ambiente inteligente se encuentran los datos ambientales relacionados con el clima. Para obtener esta información en necesario contar con datos provenientes de sensores de temperatura, humedad y luminosidad. Otro tipo de información relevante se refiere a la presencia de personas en el ambiente, para obtener la información de la entrada y salida de las personas se utilizan sensores de presencia. Ambos tipos de información son contemplados en el modelo ontológico que representa la de red de sensores Figura 4.

Según Gascón [13], un componente importante dentro de un ambiente inteligente son las redes de sensores. La ontología red de sensores tiene dos objetivos principales:

a) Representar los conceptos y propiedades de datos que permitan identificar y registrar la presencia de personas (estudiantes, empleados o visitantes) automáticamente, si están entrando o saliendo en un espacio físico específico. 
b) Representar los conceptos que permitan detectar e inferir sobre eventos simples relacionados con el medio ambiente.

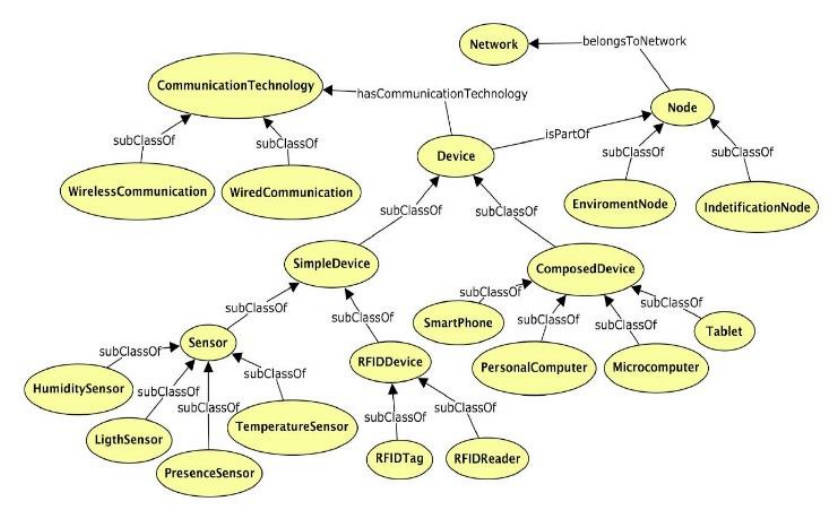

Figura 4 Ontología de red de sensores

\section{Ontología de eventos ambientales}

El modelo de eventos ambientales Figura 5, es fundamental dentro del sistema de representación, la ontología está compuesta de cuatro clases, cada una corresponde a una de las variables ambientales que se analizan en este trabajo (temperatura, humedad, luminosidad y presencia).

La primera clase contiene los incrementos o decrementos de la temperatura, la segunda corresponde a los aumentos y decrementos de la humedad, la tercera clase corresponde a las variaciones de la luminosidad y la última es la encargada de representar a los eventos de presencia.

Se compone de las clases que definen la entrada de una persona a un lugar y la salida de esta misma. Las propiedades de dato que constituyen a la ontología de eventos son hasVariation que como su nombre lo indica sirve para almacenar los valores numéricos de los cambios detectados en los eventos ambientales.

Las propiedades hasEventName y hasDescription sirven para complementar la información de los acontecimientos.

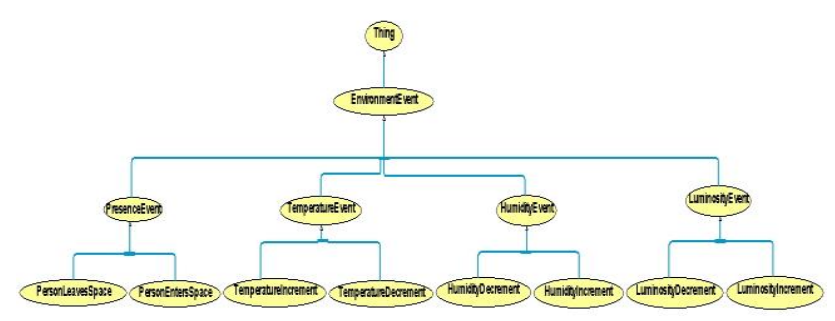

Figura 5 Ontología de eventos ambientales

\section{Sistema de Ontologías}

El resultado de la integración de las ontologías modulares presentadas es el sistema "IntelligentEnvironment" y tiene como objetivo el modelar un ambiente genérico del ámbito académico, donde participen personas con distintos roles, además de representar los espacios físicos y que cuente con una red de sensores con el fin de obtener mediciones para detectar variaciones ambientales y el momento exacto en el que ocurren.

\section{Detección de eventos ambientales usando agentes}

En este trabajo se realiza una simulación de las lecturas de datos climáticos que se generan dentro de un ambiente académico, es decir, se hace una réplica de las mediciones de temperatura, humedad, luminosidad y presencia.

Para la generación de los datos se implementaron agentes inteligentes desarrollados con el Framework Java Agent DEvelopment, o JADE, que es una plataforma de software para el desarrollo de agentes, implementada en Java.

Según Vargas-Quesada [14] un agente inteligente se define como un software desarrollado que utiliza ciertas técnicas o herramientas de inteligencia artificial, tiene como propósito percibir el entorno y actuar de una manera autónoma es decir con un grado de racionalidad e independencia.

\section{Generación de datos utilizando agentes inteligentes}

Para la generación de los datos fue necesario crear dos tipos de agentes especializados y siguen un conjunto de reglas con el fin de generar paquetes que contengan la información precisa de una lectura del medio ambiente. Para el primer conjunto "agentes ambientales", deben generar un paquete que contenga las mediciones del censado la temperatura, humedad $\mathrm{y}$ luminosidad.

En el caso de la temperatura los agentes generan mediciones aleatorias dentro de un rango de entre 13 y $26{ }^{\circ} \mathrm{C}$, porque es la temperatura promedio que se suscita en el mes de junio en la CDMX, fecha en la que se realizó la simulación. 
En el caso de la humedad se generaron datos aleatoriamente que van desde el $46 \%$ hasta el $71 \%$. Finalmente, para la luminosidad se crearon variaciones con un rango de entre $200 \mathrm{y}$ 600 luxes. Para el segundo tipo de dato "los agentes generadores de presencia" se crearon lecturas que simulan el RFIDTag que se encuentra en las tarjetas de identificación de 200 usuarios pertenecientes al modelo del contexto académico. Con estos datos los agentes realizan la simulación del comportamiento de entrada y salida en un espacio físico Figura 6.

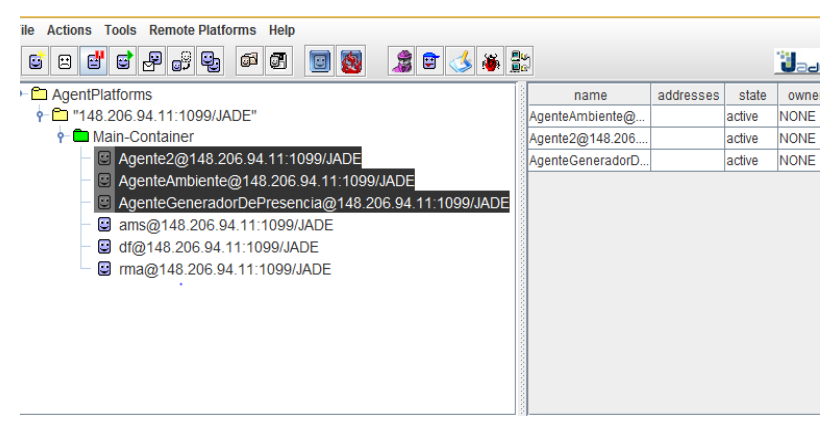

Figura 6 Simulación de datos con agentes

\section{Detección de eventos ambientales}

La secretaria del trabajo y previsión social en la NORMA Oficial Mexicana NOM-025-STPS2008, "Condiciones de iluminación en los centros de trabajo", establece los requerimientos mínimos de iluminación en las áreas de labor y centros de estudio, las cuales se pueden observar en la Tabla 1. Además, también se establece en la norma "condiciones térmicas elevadas" la temperatura poder desarrollar actividades en condiciones favorables, dentro de un ambiente académico debe oscilar entre $15^{\circ}$ y $30^{\circ} \mathrm{C}$ y la humedad relativa debería de estar entre el $50 \% \pm$ $10 \%$.

\begin{tabular}{|l|l|r|}
\hline \multicolumn{1}{|c|}{ Tarea Visual Tipo de Recinto } & \multicolumn{1}{|c|}{$\begin{array}{l}\text { Niveles de } \\
\text { Iluminación }\end{array}$} \\
\hline $\begin{array}{l}\text { Atención } \\
\text { administrativa. }\end{array}$ & $\begin{array}{l}\text { Oficinas } \\
\text { Administrativas. }\end{array}$ & 300 \\
\hline $\begin{array}{l}\text { Actividades } \\
\text { relacionadas con } \\
\text { la docencia. }\end{array}$ & $\begin{array}{l}\text { Salas de clases, } \\
\text { educación superior. }\end{array}$ & 300 \\
\hline $\begin{array}{l}\text { Distinción clara } \\
\text { de detalles: } \\
\text { captura y } \\
\text { procesamiento } \\
\text { de información. }\end{array}$ & $\begin{array}{l}\text { Talleres de precisión: } \\
\text { salas de cómputo, áreas } \\
\text { de dibujo, laboratorios. }\end{array}$ & 500 \\
\hline $\begin{array}{l}\text { Actividades } \\
\text { relacionadas con } \\
\text { la investigación. }\end{array}$ & $\begin{array}{l}\text { Salas de Profesores, } \\
\text { cubículos. }\end{array}$ \\
\hline $\begin{array}{l}\text { Lectura e } \\
\text { investigación. }\end{array}$ & Bibliotecas. & 400 \\
\hline
\end{tabular}

Tabla 1 Niveles mínimos de iluminación en un espacio académico
Con base en las normas y con la finalidad de brindar al usuario las condiciones idóneas para llevar a cabo sus actividades, se implementó un módulo de detección de eventos ambientales, que manifiesta cambios significativos en las variables de temperatura, humedad y luminosidad. Además de la identificación de personas.

El módulo de detección ambiental fue desarrollado en el lenguaje de programación Java para garantizar la perfecta comunicación con las ontologías, las cuales son codificadas utilizando el Lenguaje Web Ontológico (OWL 2.0) [15].

Para caracterizar esta parte del proyecto se definió a un evento ambiental como la diferencia entre dos lecturas de las variables ambientales antes mencionadas, y cuyo valor numérico exceda los siguientes umbrales: para la temperatura se tomó como referencia una variación a partir de $\pm 2{ }^{\circ} \mathrm{C}$. Para la humedad el umbral tomado en cuenta es de $\pm 10 \%$ y finalmente para luminosidad un evento se detecta a partir de un cambio de 50 luxes. Todos estos acontecimientos detectados por el módulo de detección son registrados en el modelo ontológico acompañados de la información complementaria: tiempo, lugar y el valor numérico de la variación.

El módulo también tiene como objetivo estar realizando periódicamente la comparación de las lecturas climáticas actuales contra los valores ideales descritos por las normas mexicanas. Si el valor actual está fuera del rango se le envía a un componente de la red de sensores "nodo actuador" una alerta para que se encargue de realizar las acciones correspondientes para regular el ambiente de manera automática, haciendo uso de sistemas de enfriamiento o calentamiento y mecanismos de iluminación.

Para el caso de la detección de presencia. Funciona con el número de identificación de una tarjeta con tecnología RFID y que se encuentra asociada con un individuo registrado dentro del modelo académico.

Con esto el sistema es capaz de identificar a la persona, además de conocer el lugar en donde se encuentra, la hora y si está entrando o saliendo de dicho espacio físico. 


\section{Instanciación de eventos ambientales en el modelo}

El poblado del modelo ontológico se lleva a cabo a través de un mecanismo de comunicación entre procesos utilizando un protocolo orientado a conexión, entre los agentes inteligentes y un servidor, que contiene al detector de eventos ambientales y el identificador de personas. Cabe mencionar que este procedimiento se realiza en un sistema concurrente donde varios procesos cooperan para poblar la ontología en el menor tiempo de cómputo posible.

En resumen, los agentes inteligentes trabajan en paralelo Figura 7, dividen el trabajo en dos tipos; los primeros se encargan de generar datos de tipo climáticos, para después nuevamente generar una división y crear subtareas para que cada uno resuelva por separado la detección de individuos de tipo: evento de temperatura, humedad, luminosidad y su clasificación (si es un evento de incremento o decremento). El segundo tipo de agentes se encargan de generar datos de presencia para crear las sub-tareas de identificación (entrada y salida de personas) que se resuelve por medio de varios ejecutores. Al final de esta etapa se obtiene una representación de conocimiento del dominio académico para ser procesable por otras aplicaciones, por ejemplo, alguna del tipo pregunta-respuesta.

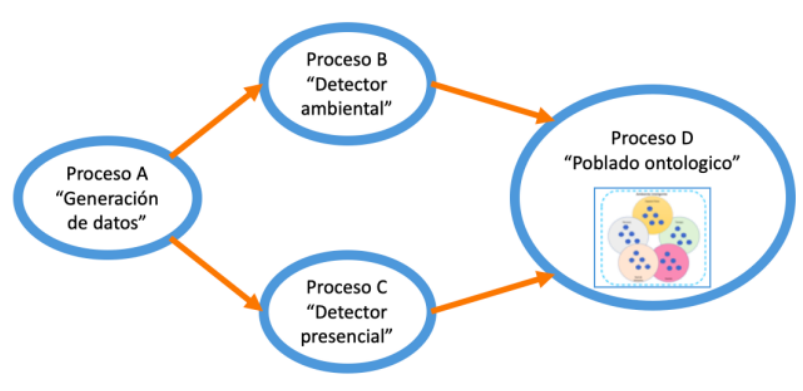

Figura 7 Procesos organizados en paralelo

\section{Resultados}

En esta sección se presenta el proceso de experimentación y los resultados al que fue sometido el detector de eventos académicos y el modelo ontológico "IntelligentEnvironment".

Para el primero se utiliza una evaluación basada en precisión, exhaustividad y valor-f. Para el segundo se utilizan escenarios de casos de uso

\section{Evaluación con las métricas: precisión y exhaustividad}

A continuación, se presentan los resultados de las métricas empleadas en la medida del rendimiento del módulo de detección de eventos ambientales. La evaluación consistió en dejar al detector evaluando los datos provenientes de los agentes inteligentes durante cinco días, para obtener un total de 9269 eventos ambientales, catalogados en: entrada y salida de personas, decremento e incremento de iluminación, humedad y temperatura.

De estos se evaluaron manualmente 750 para conocer su correctitud con la finalidad de obtener un patrón de referencia de todos los eventos detectados.

Para evaluar la precisión $P$ se utilizó la siguiente ecuación 1.

$P=\frac{\mid\{\text { eventosRelevantes }\} \cap\{\text { EventosRecuperados }\} \mid}{\mid \text { EventosRecuperados } \mid}$

Para la evaluación de la precisión se dividen los resultados en tres datos fundamentales involucrados: tiempo, persona y espacio físico. La Tabla 2 muestra los resultados obtenidos de la precisión en el proceso automático para la detección de eventos ambientales en un entorno académico.

\begin{tabular}{|l|l|l|l|l|}
\hline \multicolumn{1}{|c}{ Evento } & \multicolumn{1}{c}{ Tiempo } & Persona & \multicolumn{1}{c|}{$\begin{array}{c}\text { Espacio } \\
\text { Físico }\end{array}$} & Precisión \\
\hline $\begin{array}{l}\text { Eventos } \\
\text { ambientales }\end{array}$ & $\mathbf{0 . 9 8 4}$ & $\mathbf{0 . 9 8 6}$ & $\mathbf{0 . 9 8 3}$ & $\mathbf{0 . 9 8 4}$ \\
\hline
\end{tabular}

Tabla 2 Resultados de la precisión del detector de eventos

Como se puede observar en la evaluación de la precision para el detector ha arrojado resultados bastante favorables obteniendo un 98\% de efectividad a la hora de detectar cambios significativos en el ambiente dentro del entorno académico.

Para obtener el valor de la exhaustividad $E$ se utiliza la ecuación 2 y, además, se calculó un conjunto de datos de referencia obtenidos del producto entre la cantidad de eventos evaluados y el grado de error de cada uno de los sensores (temperatura, humedad y luminosidad), esto es conocido como el conjunto gold Standard. El grado de error fue extraído de las hojas de datos que proporcionan los fabricantes. El resultado de la exhaustividad se puede observar en la Tabla 3. 


$$
E=\frac{\mid\{\text { eventosRelevantes }\} \cap\{\text { eventosDetectados }\} \mid}{\mid \text { eventosGoldStandard } \mid}
$$

\begin{tabular}{|c|c|c|c|c|}
\hline Evento & Tiempo & Persona & $\begin{array}{l}\text { Espacio } \\
\text { Físico }\end{array}$ & $\begin{array}{c}\text { Exhaustividad } \\
\text { Total }\end{array}$ \\
\hline $\begin{array}{l}\text { Eventos } \\
\text { ambientales }\end{array}$ & 0.9035 & 0.9051 & 0.902021 & 0.90 \\
\hline
\end{tabular}

Tabla 3 Resultados de la exhaustividad del detector de eventos

Escenarios de detección de eventos ambientales

De acuerdo con Gómez [16], la evaluación de la ontología se refiere a la construcción correcta del contenido de la misma. Para la evaluación del modelo "ambiente académico inteligente" se muestran dos escenarios de casos para eventos ambientales, que sirven para valorar la representación del modelo ontológico

Escenario 1. En el salón de clases E313 se ha registrado un incremento de temperatura súbito de $2.0^{\circ} \mathrm{C}$ debido a las altas temperaturas de la zona o a la llegada masiva de individuos al lugar. El día 18 de enero de 2019 a las 19: 11:20.

En la Figura 8 se muestra un modelo con la representación de un ambiente académico, las instancias y sus respectivas relaciones descritas del texto anterior.

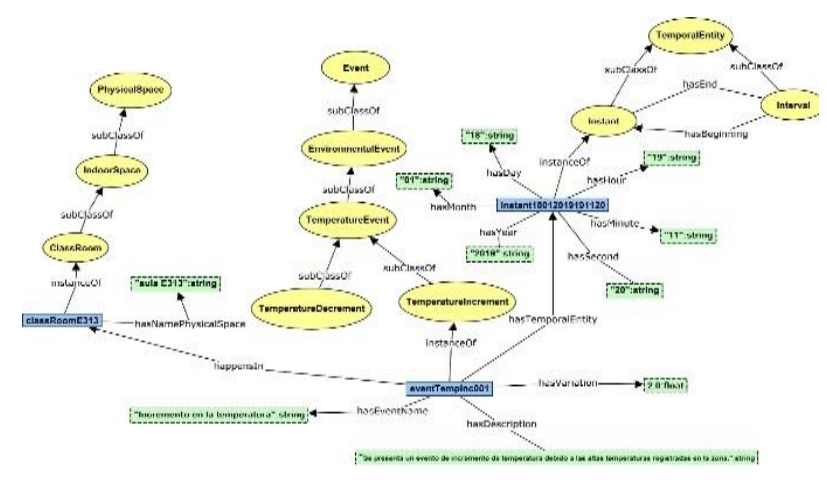

Figura 8 Escenario de casos de uso

Escenario 2. La profesora Maricela Claudia Bravo Contreras ha ingresado al cubículo H289 el día 15 de enero del 2019 a las 10:30:15 a.m.

Este escenario se representa en el modelo ontológico de ambiente inteligente como un evento de entrada, el resultado de dicha representación se muestra en el modelo ontológico, sus individuos y relaciones en la Figura 9 mostrando solamente las clases involucradas.

\section{Agradecimiento}

Este artículo fue desarrollado gracias al apoyo parcial de la SEP-PRODEP con el proyecto DSA/103.5/16/9852. Los autores también quieren agradecer a la Universidad Autónoma Metropolitana unidad Azcapotzalco.

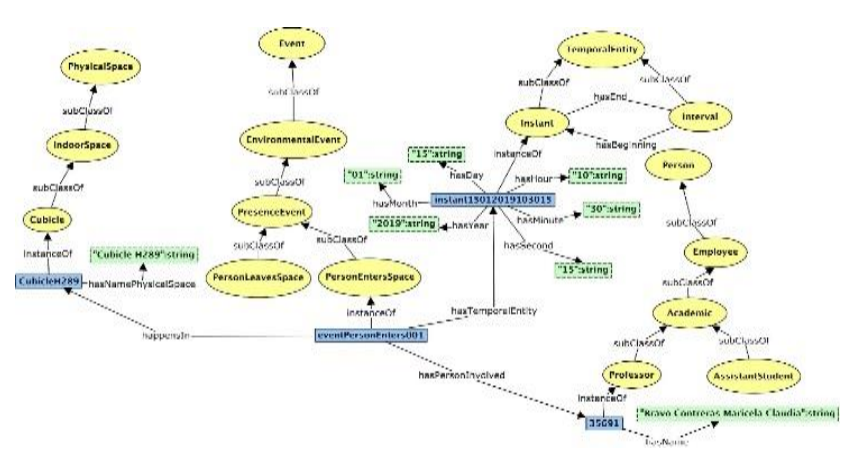

Figura 9 Escenario de casos de uso 2

\section{Conclusiones}

En este artículo se ha presentado un módulo de detección de eventos ambientales con ayuda de agentes inteligentes. Además, se describe un enfoque de solución basado en ontologías para la representación de los eventos detectados en el ambiente académico.

Con respecto a la ejecución de las pruebas y la evaluación de la funcionalidad, el rendimiento y la precisión del detector de eventos ambientales, se obtuvieron resultados bastante prometedores. La arquitectura propuesta del detector de eventos y el modelo ontológico para la representación conlleva las siguientes ventajas:

i) Posibilita conocer el estado actual de las variables ambientales: temperatura, luminosidad y humedad. De esta manera se puede ofrecer información oportuna al usuario en un ambiente sensible al contexto o para prevenir un desastre, por ejemplo, una ocurrencia de fuego no controlada.

ii) Provee la información necesaria para la identificación de individuos en un ambiente académico, con la finalidad de poder representar los eventos ambientales que suceden dentro en un espacio académico. La identificación de usuarios presentes hace que el espacio sea consciente y sensible al contexto. 
iii) Propicia la interoperabilidad entre herramientas de soporte para la especificación de un dominio académico, como pueden ser herramientas de predicción o aplicaciones de consulta. Apoyándose en el modelo ontológico desarrollado.

Los resultados preliminares obtenidos en el modelo ontológico con ejemplos de escenarios, inferencia y ha permitido concluir que la propuesta de representación de conocimiento es válida y de potencial aplicación en la investigación aplicada en las ciencias computacionales, puntualmente en el área de la inteligencia artificial.

Como trabajo a futuro, se pretende continuar extendiendo el modelo ontológico adaptándolo a las necesidades de los requerimientos de otros dominios como por ejemplo eventos relacionados con la salud.

Por otro lado, sería deseable contar con un sistema de clasificación automática de eventos utilizando las características detectadas en este trabajo.

\section{Referencias}

[1] Skocir, P. Krivic, M. Tomeljak, M. Kusek, M. \& Jezic, G. (2016). Activity detection in smart home environment. Procedia Computer Science, (96), 672-681. Recuperado de https://www.sciencedirect.com/science/article/p ii/S1877050916320609

[2] Meditskos, G., Kompatsiaris, I. (2017). iKnow: Ontology-driven situational awareness for the recognition of activities of daily living. Pervasive and Mobile Computing, 40, 1-16.

[3] Chen, L., y Nugent, C. (2009). Ontologybased activity recognition in intelligent pervasive environments. International Journal of Web Information Systems, 5(4), 410-430.

[4] Calbimonte, J. P., Ranvier, J. E., Dubosson, F., y Aberer, K. (2017). Semantic representation and processing of hypoglycemic events derived from wearable sensor data. Journal of Ambient Intelligence and Smart Environments, 9(1), 97109.
[5] Stocker, M., Rönkkö, M. \& Kolehmainen, M. (junio, 2014). Abstractions from Sensor Data with Complex Event Processing and Machine Learning. Trabajo presentado en Conference: 7th International Congress on Environmental Modelling and Software, San Diego, California.

[6] Barranco, R. (2011). IBM ISII Complex Event Processing: De la detección de eventos a la acción inmediata. Recuperado de https://www.ibm.com/developerworks/ssa/local /im/ssa/identity-insight-complex-eventprocessing/index.html

[7] Miller, G. A., Johnson-Laird, P. N. (1976). Language and perception. Belknap Press.

[8] Allen, J. F., \& Ferguson, G. (1994). Actions and events in interval temporal logic. Journal of logic and computation, 4(5), 531-579. Recuperado de https://academic.oup. com/logcom/article-

abstract/4/5/531/1042845? redirectedFrom=fullt ext

[9] Sowa, J. F. (2000). Knowledge representation: logical, philosophical, and computational foundations. Pacific Grove: Brooks/Cole.

[10] Gruber, T. R. (1995). Toward principles for the design of ontologies used for knowledge sharing? International journal of humancomputer studies, 43(5-6), 907-928.

[11] Guarino, N. (1998). Formal ontology and information systems En Guarino, N. (Ed.), Formal ontology in Information Systems (3-15).

[12] Cruz, I. (2019). Extracción y enriquecimiento de perfiles de investigación usando ontologías (Tesis de maestría). Universidad Autónoma Metropolitana Azcapotzalco, Ciudad de México.

[13] Gascón, D. (2010). Redes de sensores inalámbricos, la tecnología invisible. Bit, (180181), 53-55. Recuperado de http://www.libelium.com/libeliumdownloads/libelium-bit-coit.pdf

[14] Hípola, P. \& Vargas-Quesada, B. (1999). Agentes inteligentes: definición y tipologia. Los agentes de información. El profesional de la información, 8(4), 13-21. Recuperado de http://eprints.rclis.org/18300/ 
[15] OWL Working Group. (2009). OWL Web Ontology Language Overview. [documentación]. Recuperado de https://www.w3.org/TR/owl-features/

[16] Gómez-Pérez, A. (1996). Towards a framework to verify knowledge sharing technology. Expert Systems with Applications, 11(4), 519-529. Recuperado de https://core.ac.uk/download/pdf/11995349.pdf 